\title{
Multicomponent adhesive hard sphere models and short-ranged attractive interactions in colloidal or micellar solutions
}

\author{
Domenico Gazzillo ${ }^{\S}$, Achille Giacometti ${ }^{\S}$, Riccardo Fantoni ${ }^{\S}$, and Peter Sollich $*$ * \\ ${ }^{\S}$ Istituto Nazionale per la Fisica della Materia and Dipartimento di Chimica Fisica, \\ Università di Venezia, S. Marta DD 2137, I-30123 Venezia, Italy \\ ${ }^{\ddagger}$ King’s College London, Department of Mathematics, Strand, London WC2R 2LS, UK
}

(Dated: November 16, 2017)

We investigate the dependence of the stickiness parameters $t_{i j}=1 /\left(12 \tau_{i j}\right)$ - where the $\tau_{i j}$ are the conventional Baxter parameters - on the solute diameters $\sigma_{i}$ and $\sigma_{j}$ in multicomponent sticky hard sphere (SHS) models for fluid mixtures of mesoscopic neutral particles. A variety of simple but realistic interaction potentials, utilized in the literature to model short-ranged attractions present in real solutions of colloids or reverse micelles, is reviewed. We consider: i) van der Waals attractions, ii) hard-sphere-depletion forces, iii) polymer-coated colloids, iv) solvation effects (in particular hydrophobic bonding and attractions between reverse micelles of waterin-oil microemulsions). We map each of these potentials onto an equivalent SHS model, by requiring the equality of the second virial coefficients. The main finding is that, for most of the potentials considered, the size-dependence of $t_{i j}\left(T, \sigma_{i}, \sigma_{j}\right)$ can be approximated by essentially the same expression, i.e. a simple polynomial in the variable $\sigma_{i} \sigma_{j} / \sigma_{i j}^{2}$, with coefficients depending on the temperature $T$, or - for depletion interactions - on the packing fraction $\eta_{0}$ of the depletant particles.

PACS numbers: $64.60 .-\mathrm{i}, 82.70 . \mathrm{Dd}, 82.70 . \mathrm{Uv}$

Keywords: Sticky Hard Spheres, Mixtures, Second virial coefficient, Colloids, Micelles

*Electronic address: gazzillo@unive.it 


\section{INTRODUCTION}

Theoretical investigation of solutions of mesoscopic particles - with sizes within the range 10 - $10^{4} \AA$ - such as colloids, micelles and globular proteins, is more problematic than the study of fluids with atomic or simple molecular constituents - with sizes within the range 1-10 А [1, 2, 3, 4]. The main difficulties are due to the large difference between solute and solvent molecular sizes, as well to the possible presence of high electric charges and large charge-asymmetries. Treating mixtures of macroions and microions, with strong longranged Coulombic forces, represents a challenge for the most typical methods of the modern statistical-mechanical theory of fluids, namely Monte Carlo (MC) or molecular dynamics (MD) computer simulations and integral equations (IE) based on the Ornstein-Zernike equation coupled with approximate "closures" [5]. Large size-asymmetries entail very different time scales in MD simulations and may lead to ergodicity problems both in MC and MD calculations. Moreover, large size-differences imply several difficulties even when using IE theories.

For simplicity, the present paper will be restricted to fluids of neutral particles with spherically symmetric interactions, neglecting all Coulombic forces due to net electric charges. Starting from a fluid mixture with one or more solute species (big particles, or macroparticles) and one "solvent" species (much smaller molecules or microparticles, which might be either a true solvent or polymer coils, smaller colloidal particles, etc.), we will adopt an effective fluid approach, which eliminates all large size-asymmetries by averaging out the microscopic degrees of freedom corresponding to the solvent [6, 7]. As a consequence, the influence of the solvent is incorporated into an effective potential for the interaction between big particles, and the initial mixture is reduced to a fluid made up of only solute molecules (one or more components). Usually, at the simplest level of description the effective potential includes, in addition to a steeply repulsive part, a very short-ranged attractive one, whose range is a small fraction of the macroparticle size. Recall that a force is said to be "short-ranged" if it derives from a potential $\phi_{i j}(r)$ which vanishes as $r^{-n}$ with $n \geq 4$ when

$r \rightarrow \infty$ [8, 9]; the force $-\partial \phi_{i j} / \partial r$ then decays as $r^{-(n+1)}$. This definition of short-ranged potentials is clearly related to the second virial coefficient $B_{2, i j}$, which is a central quantity in our paper: when the forces are short-ranged in the above-mentioned sense, the integral which defines $B_{2, i j}$ (see Eq. (11) below) is finite, whereas it diverges for long-ranged interac- 
tions, i.e. when $r \leq 3$. Note that the definition of short-ranged forces is not unique in the literature. For instance, in Hirschfelder's classical reference book [10] short-range forces are the "valence or chemical forces", arising from overlap of electron clouds at very short intermolecular separations. The potential of such repulsive, and often highly directional, forces varies exponentially with the distance $r$. On the other hand, all potentials proportional to inverse powers of $r$ are called "long-ranged" by Hirschfelder [11].

Once a reasonable approximation to the effective potential is known, it could be employed in both computer simulations or IE calculations. Unfortunately, IEs can be solved analytically only in very specific cases, for some potentials and within particular "closures" [5]. The simplest model with both repulsion and attraction which is analytically tractable refers to a fluid made up of hard spheres (HS) with an infinitely narrow and infinitely deep attractive tail. This highly idealized model of adhesive or sticky hard spheres (SHS) was proposed by Baxter [12], and admits an analytical solution within the Percus-Yevick (PY) approximation [12, 13, 14]. Notwithstanding its crudeness and known shortcomings [15], the SHS model is not a purely academic exercise. In fact, it has seen continuously growing interest in the last two decades, because of its ability to describe semiquantitatively many properties of real fluids of neutral spherical particles, such as colloidal suspensions, micelles, protein solutions, microemulsions, and systems exhibiting phase transitions of several types (see for example Refs. [15, 16, 17, 18] and references therein). Accurate simulation data for one-component SHS have recently been reported by Miller and Frenkel [19].

Because of the simplicity of the SHS model, it has often been suggested to model potentials comprising a hard core and short-ranged attractive tail by means of sticky potentials. To achieve this one needs to define an appropriate equivalence between the actual interaction and its sticky representation. This mapping of a generic short-ranged potential onto a SHS interaction is usually accomplished by requiring the two different models to have equal second virial coefficients [16, 20]. Moreover, when applied to mixtures, this approach requires a further step, and this is the main point addressed in the present work.

In a series of earlier papers [21, 22, 23, 24, 25], we investigated the multi-component SHS model, focusing on its possible application to polydisperse colloidal suspensions, namely to mixtures where the number $p$ of components is so large that it can effectively be regarded as stemming from a continuous distribution. This is, for instance, the case of size polydispersity, where - in the discrete notation - a SHS mixture is fully characterized by two sets of 
parameters, i.e., the HS diameters $\left\{\sigma_{i}\right\}$ and the "stickiness" coefficients $\left\{t_{i j}=1 /\left(12 \tau_{i j}\right)\right\}$ ( $\tau_{i j}$ are Baxter's parameters); the latter depend on temperature $T$ and the strength of the interparticle adhesion. Intuitively, one expects $t_{i j}$ to depend on the diameters $\sigma_{i}$ and $\sigma_{j}$ of the interacting particles $i$ and $j$, but it is not easy to specify a priori the correct functional form, and in our previous papers we attempted some reasonably motivated choices for such a dependence.

The main purpose of the present paper is to investigate the relationship between stickiness coefficients and particles sizes, and thus to get new insights into the possible forms of the function $t_{i j}=t_{i j}\left(T, \sigma_{i}, \sigma_{j}\right)$, starting from a physically sound basis. To achieve this, we will present an overview of the most important short-ranged attractive interactions occurring in real solutions of colloids or micelles. In doing this, our claim is not to be fully exhaustive, but rather to gather sufficient physical information about the mechanisms which cause shortranged attractive interactions in solutions of mesoscopic particles, and the corresponding simplest model potentials used for their representation.

By considering several different systems - dispersion forces, depletion forces, polymercoated colloids, solvation forces (in particular, hydrophobic interactions, and reverse micelles in water-in-oil microemulsions) - we have surprisingly found strong similarities among the simplest models employed to represent this wide variety of physical phenomena. By constructing, for each of the relevant potentials, an equivalent SHS representation, we will deduce and compare the corresponding expressions for $t_{i j}=t_{i j}\left(T, \sigma_{i}, \sigma_{j}\right)$.

The paper is organized as follows. In Section II we will introduce the basic formalism, concerning the second virial coefficient, the Baxter SHS model, and the mapping rule for getting the equivalent SHS potential from a given short-ranged attraction. Sections III is dedicated to the direct van der Waals interaction, while Sections IV, V and VI survey the most important short-ranged attractions that are indirect, i.e. mediated by the solvent. The hydrophobic effect and interactions between reverse micelles will be considered in Section VI, as particular cases of solvation forces. For each model potential, a reasonable approximation to the corresponding $t_{i j}=t_{i j}\left(T, \sigma_{i}, \sigma_{j}\right)$ will be calculated. Finally, a summary, with a brief discussion, and our conclusions will be given in Section VII. 


\section{BASIC FORMALISM}

\section{A. Second virial coefficient}

For a multi-component fluid, the second virial coefficient of the osmotic pressure reads $B_{2}=\sum_{i, j} x_{i} x_{j} B_{2, i j}$, where $x_{i}$ is the molar fraction of species $i$, and the partial second virial coefficient for the $i-j$ interaction is given by

$$
B_{2, i j}=-\frac{1}{2} \int f_{i j}(r) d \mathbf{r}=-2 \pi \int_{0}^{+\infty} f_{i j}(r) r^{2} d r,
$$

with

$$
f_{i j}(r)=\exp \left[-\beta \phi_{i j}(r)\right]-1
$$

being the Mayer function, $\beta=\left(k_{B} T\right)^{-1}, k_{B}$ the Boltzmann constant, and $T$ the absolute temperature.

When the actual potential consists of a hard core plus a short-ranged attractive tail, i.e., $\phi_{i j}(r)=\phi_{i j}^{\mathrm{HS}}(r)+\phi_{i j}^{\mathrm{tail}}(r)$, one gets

$$
\begin{gathered}
B_{2, i j}=B_{2, i j}^{\mathrm{HS}}+B_{2, i j}^{\mathrm{tail}} \\
B_{2, i j}^{\mathrm{tail}}=-2 \pi \int_{\sigma_{i j}}^{+\infty} f_{i j}^{\mathrm{tail}}(r) r^{2} d r=B_{2, i j}^{\mathrm{HS}}\left[-3 \int_{1}^{+\infty} f_{i j}^{\mathrm{tail}}\left(\sigma_{i j} x\right) x^{2} d x\right],
\end{gathered}
$$

where $\sigma_{i}$ is the HS diameter for particles of species $i$ and we set $\sigma_{i j}=\left(\sigma_{i}+\sigma_{j}\right) / 2$ as usual, introducing also the shorthands $B_{2, i j}^{\mathrm{HS}}=(2 \pi / 3) \sigma_{i j}^{3}$ and $f_{i j}^{\text {tail }}(r)=\exp \left[-\beta \phi_{i j}^{\text {tail }}(r)\right]-1$.

Often, the required integration cannot be performed analytically, but if $\phi_{i j}^{\text {tail }}(r)$ is sufficiently small compared to the thermal energy $k_{B} T$, then approximate analytical expressions may be obtained after expanding the Mayer function $f_{i j}^{\text {tail }}(r)$ in powers of $Y \equiv-\beta \phi_{i j}^{\text {tail }}(r)$. A numerical estimate of the range of applicability and the maximum relative error $\Delta_{\max }=$ $\max \left|1-f_{\text {approx }} / f\right|$, for each of the three simplest approximations, is

$$
f=e^{Y}-1 \approx\left\{\begin{array}{ccc}
Y & 0<Y \lesssim 0.1 & \Delta_{\max } \sim 5 \% \\
Y+Y^{2} / 2 & 0<Y \lesssim 0.6 & \Delta_{\max } \sim 5 \% \\
Y+Y^{2} / 2+Y^{3} / 6 & 0<Y \lesssim 1 & \Delta_{\max } \lesssim 3 \%
\end{array}\right.
$$

\section{B. Adhesive hard spheres as a limiting case of square-well model}

Probably, the simplest two-parameter representation of a spherically symmetric interaction with steeply repulsive core and short-ranged attractive tail is the square-well (SW) 
potential

$$
\phi_{i j}^{\mathrm{SW}}(r)= \begin{cases}+\infty & 0<r<\sigma_{i j} \\ -\epsilon_{i j} & \sigma_{i j} \leq r \leq \sigma_{i j}+w_{i j} \\ 0 & r>\sigma_{i j}+w_{i j}\end{cases}
$$

with $\epsilon_{i j}>0$ and $w_{i j}$ being the depth and width of the well, respectively. The corresponding partial second virial coefficient reads

$$
\begin{aligned}
B_{2, i j}^{\mathrm{SW}} & =B_{2, i j}^{\mathrm{HS}}\left\{1-\left(e^{\beta \epsilon_{i j}}-1\right)\left[\left(1+\Delta_{i j}\right)^{3}-1\right]\right\} \\
& =B_{2, i j}^{\mathrm{HS}}\left[1-3\left(e^{\beta \epsilon_{i j}}-1\right)\left(\Delta_{i j}+\Delta_{i j}^{2}+\frac{1}{3} \Delta_{i j}^{3}\right)\right]
\end{aligned}
$$

with $\Delta_{i j}=w_{i j} / \sigma_{i j} \geq 0$. Eq. (17) shows that, if the well is narrow $\left(\Delta_{i j} \ll 1\right), B_{2, i j}^{\mathrm{SW}}$ can be significantly different from $B_{2, i j}^{\mathrm{HS}}$ only when the attraction is strong enough $\left(e^{\beta \epsilon_{i j}} \gg 1\right)$.

Unfortunately, despite the simplicity of the SW model, no satisfactory analytical solution of the resulting IEs has been found so far. However, such a solution can be found within the Percus-Yevick (PY) approximation for a special limiting case, when the well width $\Delta_{i j}$ goes to zero but the depth $\epsilon_{i j}$ goes to infinity in such a way that the contribution of the attraction to the second virial coefficient remains finite and different from zero (Baxter's sticky limit) [12]. The short-ranged attraction becomes a surface adhesion, and the particles of the resulting model are thus named adhesive or sticky hard spheres. From Eq. (7) one sees that Baxter's condition on $B_{2, i j}^{\mathrm{SW}}$ requires the product $\left(e^{\beta \epsilon_{i j}}-1\right) \Delta_{i j} \equiv t_{i j}$ to be independent of $\Delta_{i j}$ for small $\Delta_{i j}$, and this leads to the following condition for the SW depth

$$
\epsilon_{i j}^{\text {Baxter SW }}=k_{B} T \ln \left(1+\frac{t_{i j}}{\Delta_{i j}}\right) .
$$

As previously mentioned, our $t_{i j}$ is simply related to Baxter's original parameter $\tau_{i j}$ by

$$
t_{i j}=\frac{1}{12 \tau_{i j}} \geq 0
$$

Here, $t_{i j}$ measures the strength of surface adhesiveness or "stickiness" between particles of species $i$ and $j$, and must be an unspecified decreasing function of $T$. In fact, as $T \rightarrow \infty$ one must also have $\tau_{i j} \rightarrow \infty$, in order to recover the correct HS limit. The SHS models must therefore satisfy the high-temperature condition

$$
\lim _{T \rightarrow \infty} t_{i j}=0
$$


A consequence of Eq. (8) is a very simple expression for the SW Mayer function

$$
f_{i j}^{\text {Baxter SW }}(r)= \begin{cases}-1 & 0<r<\sigma_{i j}, \\ t_{i j} \sigma_{i j} / w_{i j} & \sigma_{i j} \leq r \leq \sigma_{i j}+w_{i j}, \\ 0 & r>\sigma_{i j}+w_{i j},\end{cases}
$$

Baxter focused on $f_{i j}$, since this quantity directly determines $B_{2, i j}$ and, furthermore, the coefficients in the cluster expansion of thermodynamic properties and correlation functions can be expressed in terms of multi-dimensional integrals of products of Mayer functions [5]. The simple functional form of $f_{i j}$ Baxter SW $(r)$ then allows one to calculate analytically many quantities of interest. In the "sticky limit" $\left\{w_{i j}\right\} \rightarrow\{0\}$, the Mayer function becomes

$$
f_{i j}^{\mathrm{SHS}}(r)=\left[\theta\left(r-\sigma_{i j}\right)-1\right]+t_{i j} \sigma_{i j} \delta_{+}\left(r-\sigma_{i j}\right)
$$

with $\theta(x)$ being the Heaviside function ( $=0$ when $x<0$, and $=1$ when $x>0$ ) and $\delta_{+}(x)$ an asymmetric Dirac distribution [26], while the SHS second virial coefficient is simply

$$
B_{2, i j}^{\mathrm{SHS}}=B_{2, i j}^{\mathrm{HS}}\left(1-3 t_{i j}\right)
$$

\section{Mapping onto equivalent SHS model}

On comparing Eqs. (13) and (44), one has

$$
t_{i j}^{\mathrm{eq}(\mathrm{tail})}=-\frac{B_{2, i j}^{\mathrm{tail}}}{3 B_{2, i j}^{\mathrm{HS}}}
$$

and hence the following mapping rule: the parameters $t_{i j}$ of the equivalent SHS model must be given by

$$
t_{i j}^{\mathrm{eq}(\mathrm{tail})}=\frac{1}{\sigma_{i j}^{3}} \int_{\sigma_{i j}}^{+\infty} f_{i j}^{\mathrm{tail}}(r) r^{2} d r=\int_{1}^{+\infty} f_{i j}^{\mathrm{tail}}\left(\sigma_{i j} x\right) x^{2} d x .
$$

This is the main relation used in the remaining part of the paper. The superscript in $t_{i j}^{\text {eq(tail) }}$ means: this $t_{i j}$ yields the SHS potential equivalent to $\phi_{i j}^{\text {tail }}$.

\section{VAN DER WAALS ATTRACTION}

The main direct attraction between two neutral molecules $i$ and $j$ is the van der Waals $(\mathrm{vdW})$ interaction, represented by the potential $\phi_{i j}^{\mathrm{vdW}}(r)=-C_{i j}^{\mathrm{vdW}} r^{-6}$, which is - in general 
- the sum of three different contributions. For most simple molecules - except the small highly polar ones - the vdW attraction is almost exclusively determined by the dispersion forces; the latter are in fact the only contribution to the vdW forces if both molecules are nonpolar.

\section{A. Dispersion forces}

The dispersion or London forces are induced-dipole/induced-dipole interactions, whose potential is given by the London formula [10]

$$
\phi_{i j}^{\text {disp }}(r)=-\frac{C_{i j}}{r^{6}}, \quad C_{i j}=\frac{3}{2} \frac{I_{i} I_{j}}{I_{i}+I_{j}} \alpha_{i}^{\prime} \alpha_{j}^{\prime}, \quad \text { for large } r,
$$

where $I_{i}$ and $\alpha_{i}^{\prime}$ are, respectively, the ionization energy and polarizability volume for molecules of species $i$. As the name suggests, $\alpha_{i}^{\prime}$ has the dimensions of volume. It can also be written as $\alpha_{i} /\left(4 \pi \varepsilon_{0}\right)$, where $\varepsilon_{0}$ is the permittivity of the vacuum and $\alpha_{i}$ is the polarizability of species $i$, which increases with increasing molecular size and number of electrons. Hence the polarizability volume is proportional to the molecular volume, i.e., $\alpha_{i}^{\prime} \propto \sigma_{i}^{3}$.

This polarizability effect alone can produce considerable molecular attraction, and is responsible for the formation of liquid phases from gases of nonpolar substances (argon, hydrogen, nitrogen, etc.). The name "dispersion forces" stems from the fact that the electronic oscillations producing the London attraction are also responsible for the dispersion of light.

\section{B. Hamaker's macroscopic approximation}

Colloids, micelles and globular proteins are mesoscopic particles formed by a very large number of polarizable molecules (typically $10^{10}$ in micrometer-sized particles) [1]. As a consequence, the total attraction energy between such macroparticles can be obtained by pairwise summation of London energies between all molecules of the two interacting bodies. Hamaker [27] performed an approximate calculation [2] for the energy of interaction of two fully macroscopic bodies $i$ and $j$ in a vacuum, with densities $\rho_{i}$ and $\rho_{j}$ and occupying volumes $V_{i}$ and $V_{j}$. Replacing the discrete distribution of molecules inside each body with a 
continuous one, Hamaker obtained for two spheres of arbitrary size [2]

$$
\begin{aligned}
\phi_{i j}^{\mathrm{H}}(r) & =-\frac{A_{i j}^{\mathrm{H}}}{12}\left[\frac{\sigma_{i} \sigma_{j}}{r^{2}-\sigma_{i j}^{2}}+\frac{\sigma_{i} \sigma_{j}}{r^{2}-L_{i j}^{2}}+2 \ln \left(\frac{r^{2}-\sigma_{i j}^{2}}{r^{2}-L_{i j}^{2}}\right)\right] \\
& =-\frac{A_{i j}^{\mathrm{H}}}{12}\left[\frac{\sigma_{i} \sigma_{j}}{r^{2}}\left(\frac{1}{1-\sigma_{i j}^{2} / r^{2}}+\frac{1}{1-L_{i j}^{2} / r^{2}}\right)+2 \ln \left(\frac{1-\sigma_{i j}^{2} / r^{2}}{1-L_{i j}^{2} / r^{2}}\right)\right]
\end{aligned}
$$

where $L_{i j}=\left|\sigma_{i}-\sigma_{j}\right| / 2$, and $\sigma_{i j}<r<+\infty$. Here, $A_{i j}^{\mathrm{H}}=\pi^{2} \rho_{i} \rho_{j} C_{i j}$ [4] is referred to as Hamaker's constant, and has dimensions of energy. As $C_{i j} \propto \alpha_{i}^{\prime} \alpha_{j}^{\prime} \propto \sigma_{i}^{3} \sigma_{j}^{3}$, and $\rho_{i} \rho_{j} \propto$ $\sigma_{i}^{-3} \sigma_{j}^{-3}, A_{i j}^{\mathrm{H}}$ is nearly independent of $i$ and $j$. In the case where all mesoscopic particles are made up of the same material but have different diameters (discrete size polydispersity) $A_{i j}^{\mathrm{H}}$ reduces to $A_{\mathrm{H}}=\pi^{2} \rho^{2} C$, which is a property of the material itself.

Hamaker's macroscopic result has also been applied to mesoscopic particles, with the justification that the potential (17) has a scaling property: if $r, \sigma_{i}, \sigma_{j}$ are all multiplied by a factor $\gamma$, the attraction energy remains unaltered, i.e., $\phi_{i j}^{\mathrm{H}}\left(\gamma r, \gamma \sigma_{i}, \gamma \sigma_{j}\right)=\phi_{i j}^{\mathrm{H}}\left(r, \sigma_{i}, \sigma_{j}\right)$. Note, however, that Hamaker's formula refers to two spheres in free space, i.e., it neglects the screening of London forces due to the suspending medium.

In the limit $r \rightarrow+\infty$, a series expansion of Eq. (17) yields

$$
\phi_{i j}^{\mathrm{H}}(r) \approx-\frac{A_{i j}^{\mathrm{H}}}{36} \frac{\sigma_{i}^{3} \sigma_{j}^{3}}{r^{6}}, \quad \text { for } r \gg \sigma_{i j}>L_{i j},
$$

which means that at large distances the two spheres behave, to leading order, like pointparticles even though the factors $\sigma_{i}^{3}$ and $\sigma_{j}^{3}$ stem from HS volumes.

On the other hand, the Hamaker potential is singular at contact, i.e., when $r \rightarrow \sigma_{i j}$. This is due to the approximation of regarding the two spheres as continuous distributions of point-particles, neglecting all intermolecular repulsions. The leading divergence is

$$
\phi_{i j}^{\mathrm{H}}(r) \approx-\frac{A_{i j}^{\mathrm{H}}}{12} \frac{\sigma_{i} \sigma_{j}}{r^{2}-\sigma_{i j}^{2}} \approx-\frac{A_{i j}^{\mathrm{H}}}{24} \frac{\sigma_{i} \sigma_{j}}{\sigma_{i j}} \frac{1}{r-\sigma_{i j}} \quad \text { for } \quad 0<r-\sigma_{i j} \ll \min \left(\sigma_{i}, \sigma_{j}\right) .
$$

This divergence simply means that the continuum picture must break down and molecular granularity - with excluded-volume effects - cannot be neglected once the closest distance $r-\sigma_{i j}$ between the two spherical surfaces becomes very small.

Such a deep attractive potential would lead to irreversible association or "flocculation" of the suspended particle. This effect can be avoided in one of two different ways, namely by charge stabilization or steric stabilization. In the first case, some surface chemical groups 
of the particles become partially ionized in water, and the resulting electrostatic repulsion makes close contact impossible. In the second case, stabilization is achieved by grafting polymer chains ("hair") to the particle surfaces. Both stabilization mechanisms - extensively used for colloidal suspensions - imply that the closest approach distance between $i$ and $j$ becomes larger than $\sigma_{i j}$, i.e., $\sigma_{i j}^{\text {eff }}=\sigma_{i j}+\delta$, with $\delta>0$ being an additional characteristic length. The Hamaker singularity at contact is thus avoided, and the vdW attraction may then be treated as a small perturbation, if the effective HS diameter is sufficiently large compared to the bare one (in sterically stabilized colloidal suspensions, $\sigma^{\text {eff }}$ exceeds $\sigma$ typically by 10\%). Moreover, it is possible to strongly reduce the value of the Hamaker constant by "refractive index matching" [2].

A numerical estimate of the strength of the Hamaker attraction is given - in the onecomponent case, for simplicity - by the quantity

$$
Y_{\max } \equiv-\beta \phi^{\mathrm{H}}(\sigma+\delta)=3 H(1+\lambda) \frac{T_{\mathrm{H}}}{T},
$$

where $\lambda \equiv \delta / \sigma$,

$$
H(u)=\frac{1}{u^{2}-1}+\frac{1}{u^{2}}+2 \ln \left(1-\frac{1}{u^{2}}\right)
$$

and, from Eq. (18), we have defined a Hamaker temperature as

$$
T_{\mathrm{H}}=\frac{A_{\mathrm{H}}}{36 k_{B}}
$$

which depends on the material which constitutes the particles. In most cases, $A_{\mathrm{H}}$ lies between $10^{-20}$ and $10^{-19}$ J, i.e., $2 k_{B} T \lesssim A_{\mathrm{H}} \lesssim 20 k_{B} T$, where $T=298.15 \mathrm{~K}$. A typical value $A_{\mathrm{H}}=0.5 \times 10^{-20} \mathrm{~J}\left(=10 k_{B} T\right)$ yields $T_{\mathrm{H}}=100 \mathrm{~K}$, and thus - at room temperature -

$$
\phi^{\mathrm{H}}(\sigma+\delta) \approx \begin{cases}-2 k_{B} T & \text { if } \lambda=0.1 \\ -0.6 k_{B} T & \text { if } \lambda=0.2 \\ -0.2 k_{B} T & \text { if } \lambda=0.3\end{cases}
$$

Using Eq. (20) for $Y_{\max }$ together with the criteria in Eq. (5), one finds the approximate lower bound $T^{\mathrm{min}} / \mathrm{K}$ for the applicability, respectively, of the linear, quadratic and cubic approximations to the Mayer function, as reported in Table —

Thus, whereas the linear approximation works only at high temperatures, the quadratic one is already sufficient even at room temperature if $\lambda \gtrsim 0.2$. 
Unfortunately, analytical integration of the expression (17) is not possible, and consequently no result for $t_{i j}^{\mathrm{eq}(\mathrm{H})}$ can be obtained directly from $\phi_{i j}^{\mathrm{H}}(r)$. Nevertheless, in order to get a rough approximation to $t_{i j}^{\mathrm{eq}(\mathrm{H})}$, we propose an analytically integrable interpolation of the correct behavior of $\phi_{i j}^{\mathrm{H}}(r)$ at short and large distances, i.e.

$$
\phi_{i j}^{\mathrm{H}-\text { interp }}(r)=-\frac{A_{i j}^{\mathrm{H}}}{36}\left\{\frac{3}{2} \frac{\sigma_{i} \sigma_{j}}{\sigma_{i j}} \frac{1}{r-\sigma_{i j}} \exp \left(-\frac{r-\sigma_{i j}}{L}\right)+\frac{\sigma_{i}^{3} \sigma_{j}^{3}}{r^{6}}\left[1-\exp \left(-\frac{r-\sigma_{i j}}{L}\right)\right]\right\},
$$

where $\sigma_{i j}+\delta \leq r<+\infty$, and $L$ acts as a screening length. When $\delta \simeq 0.1 \sigma, L=0.108 \sigma \approx \delta$ yields a satisfactory contact value, i.e., $\phi_{i j}^{\mathrm{H}-\text { interp }}\left(\sigma_{i j}+\delta\right) \approx \phi_{i j}^{\mathrm{H}}\left(\sigma_{i j}+\delta\right)$. Using the linear approximation - valid at high temperatures - one gets

$$
t_{i j}^{\mathrm{eq}(\mathrm{H}-\mathrm{interp})} \approx \frac{1}{k_{B} T} \frac{A_{i j}^{\mathrm{H}}}{24} \frac{\sigma_{i} \sigma_{j}}{\sigma_{i j}^{2}}\left[E_{1}\left(\frac{\delta}{L}\right)+2 e^{-\delta / L} \frac{L}{\sigma_{i j}}+\left(1+\frac{\delta}{L}\right) e^{-\delta / L}\left(\frac{L}{\sigma_{i j}}\right)^{2}\right],
$$

where $E_{1}(z)=\int_{z}^{+\infty} \frac{e^{-u}}{u} d u$ is the exponential integral. However, since the factors $L / \sigma_{i j}^{n} \approx$ $\delta / \sigma_{i j}^{n}$ refer to big particles, the leading term - at least within the linear approximation - is

$$
t_{i j}^{\mathrm{eq}(\mathrm{H}-\mathrm{interp})} \approx \frac{3}{2} E_{1}\left(\frac{\delta}{L}\right)\left(\frac{T_{\mathrm{H}}}{T} \frac{\sigma_{i} \sigma_{j}}{\sigma_{i j}^{2}}\right) .
$$

\section{Polarizable hard spheres. Sutherland model}

Focusing only on the $r^{-6}$ part of the Hamaker potential, which represents the longdistance polarizability, one could define a simpler model, corresponding to a mixture of mesoscopic HS with dispersion attractions, called polarizable hard spheres (PHS), i.e.,

$$
\phi_{i j}^{\mathrm{PHS}}(r)=\left\{\begin{array}{cl}
+\infty, & 0<r<\sigma_{i j} \\
-A_{i j} \sigma_{i}^{3} \sigma_{j}^{3} / r^{6} & r \geq \sigma_{i j}
\end{array}\right.
$$

where the choice

$$
A_{i j}=\frac{A_{i j}^{\mathrm{H}}}{36}
$$

ensures the mesoscopic size of the particles. If all particles are made up of the same material substance, then $\phi_{i j}^{\mathrm{PHS}}(r)=-A \sigma_{i}^{3} \sigma_{j}^{3} / r^{6}$ for $r \geq \sigma_{i j}$.

The potential (25) may be regarded as a special case of the Sutherland model, which represents rigid spheres which attract one another according to an inverse-power law, i.e., $\phi^{\text {Sutherland }}(r)=-\epsilon(\sigma / r)^{b}$ for $r \geq \sigma \quad(\epsilon>0)$ [10]. Indeed, one could rewrite it as $\phi_{i j}^{\mathrm{PHS}}(r)=$ $-\epsilon_{i j}^{\mathrm{PHS}}\left(\sigma_{i j} / r\right)^{6}$, with $\epsilon_{i j}^{\mathrm{PHS}}=A\left(\sigma_{i} \sigma_{j} / \sigma_{i j}^{2}\right)^{3}$ for particles with the same material composition. 
The strength of this interaction - in the one-component case - is then given by

$$
Y_{\max } \equiv-\beta \phi^{\mathrm{PHS}}(\sigma)=\frac{T_{\mathrm{H}}}{T} \approx \frac{100 \mathrm{~K}}{T}
$$

after taking $T_{\mathrm{H}} \approx 100 \mathrm{~K}$. From the results outlined in Eq. (5), a linearization of the Mayer function makes sense for $T \gtrsim 1000 \mathrm{~K}$. A quadratic approximation is feasible when $T \gtrsim 167 \mathrm{~K}$. Finally, the cubic approximation holds for $T \gtrsim 100 \mathrm{~K}$.

Therefore, in the multi-component case, one can safely adopt the cubic approximation to $f_{i j}^{\mathrm{PHS}}(r)$ and perform the integration in Eq. (15), obtaining

$$
t_{i j}^{\mathrm{eq}(\mathrm{PHS})}=12 \frac{T_{\mathrm{H}}}{T}\left(\frac{\sigma_{i} \sigma_{j}}{\sigma_{i j}^{2}}\right)^{3}+15\left[\frac{T_{\mathrm{H}}}{T}\left(\frac{\sigma_{i} \sigma_{j}}{\sigma_{i j}^{2}}\right)^{3}\right]^{2}+8\left[\frac{T_{\mathrm{H}}}{T}\left(\frac{\sigma_{i} \sigma_{j}}{\sigma_{i j}^{2}}\right)^{3}\right]^{3} .
$$

In the one-component case this expression reduces to

$$
t^{\mathrm{eq}(\mathrm{PHS})}=12 \frac{T_{\mathrm{H}}}{T}+15\left(\frac{T_{\mathrm{H}}}{T}\right)^{2}+8\left(\frac{T_{\mathrm{H}}}{T}\right)^{3},
$$

For $T_{\mathrm{H}} \approx 100 \mathrm{~K}$, this results - at $T \approx 300 \mathrm{~K}-$ in a value $t^{\mathrm{eq}(\mathrm{PHS})} \simeq 5.96$, which corresponds in Baxter's parametrization to

$$
\tau=\frac{1}{12 t} \simeq 0.014
$$

and lies well below the critical temperature of the SHS fluid, $\tau_{c}=0.1133 \pm 0.0005\lfloor 19]$.

\section{EXCLUDED VOLUME DEPLETION FORCES}

In general, the indirect, solvent-mediated, solute-solute interactions depend on both the solute-solvent and solvent-solvent ones, and thus may be very difficult to evaluate [6, 7, 20, 28, 29]. We will now report several very simplified cases.

Asakura and Oosawa (AO) [30], and independently Vrij [31], first showed that two big (colloidal, or solute) particles, immersed in a sea of small particles, feel a mutual attraction when their surfaces get closer than the size of the smaller particles (depletion attraction). This effect is an indirect attraction originating from the interactions of the two big particles with the small ones of the environment, even if these latter consist of, say, hard spheres. In mixtures with neutral components, the small particles - hereafter referred to also as depletant particles - may correspond, for example, to solvent molecules, non-adsorbing polymer coils, or smaller colloidal particles. 
Upon adding, for instance, polymers to a stable colloidal suspension, the colloidal particles tend to aggregate. The polymer-induced depletion forces between the colloidal particles can cause formation of colloidal crystals or flocculation.

In the $\mathrm{AO}$ model, originally designed to describe colloid-polymer mixtures, the big-big (colloid-colloid) interactions as well as the big-small ones are modeled as excluded-volume HS interactions, while the small-small interactions are assumed to be zero (ideal gas approximation, corresponding to mutually interpenetrable, non-interacting depletant molecules). In particular, polymer coils are assumed to have an effective HS diameter equal to twice their radius of gyration.

Consider two big HS of species $i$ and $j$ at distance $r$, with radii $R_{i}$ and $R_{j}$, in a dilute suspension of depletant spheres of species 0 , with radius $R_{0}$. The solute molecule $i$ produces a spherical excluded-volume region of radius $\sigma_{i 0}=R_{i}+R_{0}$ around itself where the centers of the depletant particles cannot penetrate; this is also called the depletion zone. When the shortest distance $r-\left(R_{i}+R_{j}\right)=r-\sigma_{i j}$ between the surfaces of $i$ and $j$ becomes less than the diameter $\sigma_{0}=2 R_{0}$, the two depletion spheres surrounding $i$ and $j$ overlap and the small particles are expelled from the region between the big molecules. This implies that the thermal impact forces on the pair $i$ and $j$ from the "outside" are only partially compensated by those from the "inside" (see Fig. 8 of Ref. [31]). The depletion effect is due to this unbalanced pressure difference, which pushes the big particles together, resulting in a net attraction. From another point of view, the overlapping of excluded volumes implies that the total free volume accessible to small particles increases, leading to a gain in the system entropy with a consequent decrease of the Gibbs free energy. This trend to decrease free energy produces an effective indirect attraction between the big spheres. AO and Vrij [30, 31] evaluated the HS-depletion (HS-depl) potential as

$$
\begin{gathered}
\phi_{i j}^{\text {HS-depl }}(r)=\left\{\begin{array}{cc}
+\infty & 0<r<\sigma_{i j} \\
-\rho_{0} k_{B} T V_{i j}^{\text {overlap }}(r) & \sigma_{i j} \leq r \leq D_{i j} \\
0 & r>D_{i j},
\end{array}\right. \\
V_{i j}^{\text {overlap }}(r)=\frac{\pi}{12} \frac{1}{r}\left(D_{i j}-r\right)^{2}\left[3 D_{i i} D_{j j}-4 D_{i j}\left(D_{i j}-r\right)+\left(D_{i j}-r\right)^{2}\right]
\end{gathered}
$$

where $\rho_{0}$ is the number density of the depletant molecules, $D_{i j} \equiv \sigma_{i j}+\sigma_{0}$, and $V_{i j}^{\text {overlap }}(r)$ denotes the lens-shaped overlap volume of two spheres with radii $\sigma_{i 0}=\left(\sigma_{i}+\sigma_{0}\right) / 2$ and $\sigma_{0 j}=\left(\sigma_{j}+\sigma_{0}\right) / 2$, at distance $r$ (see Appendix). The attraction increases linearly with 
temperature and with the concentration of depletant particles. Since the AO model includes only HS interactions, the corresponding depletion forces have a purely entropic origin. Finally, it should be emphasized that the $\mathrm{AO}$ approximation is valid only for dilute suspensions of depletant molecules, i.e., at low $\rho_{0}$ values; formally, this last restriction can be removed by replacing $\rho_{0}$ by the density of polymer in a large reservoir connected to the system.

The tail of $\phi_{i j}^{\mathrm{HS}-\mathrm{depl}}(r)$ has a finite range, equal to the diameter $\sigma_{0}$ of the depletant molecules. The attraction strength can be estimated from

$$
Y_{i j, \max } \equiv-\beta \phi_{i j}^{\mathrm{HS}-\mathrm{depl}}\left(\sigma_{i j}\right)=\eta_{0}\left(1+\frac{3}{2} \frac{\sigma_{i} \sigma_{j}}{\sigma_{i j}} \frac{1}{\sigma_{0}}\right),
$$

where $\eta_{0}=(\pi / 6) \rho_{0} \sigma_{0}^{3}$ is the packing fraction of the depletant particles. Note that $Y_{i j, \max }$ does not depend on temperature, but the attraction strength may be tuned by varying $\eta_{0}$.

For one-component solutes, $Y_{i j, \max }$ reduces to $Y_{\max }=\eta_{0}(1+1.5 / \lambda)$ with $\lambda \equiv \sigma_{0} / \sigma$. In this case - following again the criteria given in Eq. (5) - the upper boundary $\eta_{0}^{\max }$ for the applicability of the linear, quadratic and cubic approximations to the Mayer function given as a function of $\lambda$, respectively, is reported in Table II

The quadratic approximation result for the equivalent SHS model is

$$
\begin{aligned}
t_{i j}^{\mathrm{eq}(\mathrm{HS}-\mathrm{depl})} \approx & \frac{\eta_{0}}{2}\left[\frac{\sigma_{i} \sigma_{j}}{\sigma_{i j}^{2}}+\left(1+\frac{1}{4} \frac{\sigma_{i} \sigma_{j}}{\sigma_{i j}^{2}}\right) \lambda_{i j}+\frac{1}{2} \lambda_{i j}^{2}+\frac{1}{12} \lambda_{i j}^{3}\right] \\
& +\frac{1}{10}\left(\frac{\eta_{0}}{2}\right)^{2}\left[9\left(\frac{\sigma_{i} \sigma_{j}}{\sigma_{i j}^{2}}\right)^{2} \frac{1}{\lambda_{i j}}+16 \frac{\sigma_{i} \sigma_{j}}{\sigma_{i j}^{2}}+\frac{4}{7}\left(\frac{13}{7}+4 \frac{\sigma_{i} \sigma_{j}}{\sigma_{i j}^{2}}\right) \lambda_{i j}+\frac{17}{7} \lambda_{i j}^{2}+\frac{17}{63} \lambda_{i j}^{3}\right]
\end{aligned}
$$

where $\lambda_{i j} \equiv \sigma_{0} / \sigma_{i j}$. As remarked, this expression does not depend on $T$, since the solutesolvent interactions are of purely HS character. If $\lambda_{i j} \ll 1$, then the leading terms are

$$
t_{i j}^{\mathrm{eq}(\mathrm{HS}-\mathrm{depl} \mathrm{l})} \approx\left[\frac{\eta_{0}}{2}+\frac{8}{5}\left(\frac{\eta_{0}}{2}\right)^{2}\right]\left(\frac{\sigma_{i} \sigma_{j}}{\sigma_{i j}^{2}}\right)+\frac{9}{10}\left(\frac{\eta_{0}}{2}\right)^{2} \frac{1}{\lambda_{i j}}\left(\frac{\sigma_{i} \sigma_{j}}{\sigma_{i j}^{2}}\right)^{2} .
$$

Generalizing from the form of the quadratic approximation for general $\lambda_{i j}$, one expects the cubic approximation to yield a result of the form

$$
t_{i j}^{\mathrm{eq}(\mathrm{HS}-\mathrm{depl})} \approx C_{1}\left(\eta_{0} \frac{\sigma_{i} \sigma_{j}}{\sigma_{i j}^{2}}\right)+C_{2}\left(\eta_{0} \frac{\sigma_{i} \sigma_{j}}{\sigma_{i j}^{2}}\right)^{2}+C_{3}\left(\eta_{0} \frac{\sigma_{i} \sigma_{j}}{\sigma_{i j}^{2}}\right)^{3} .
$$

Several other studies of depletion forces, which go beyond the entropic HS approach by taking into account more refined representions of the solute-solvent and solvent-solvent interactions, are also available in the recent literature [6, 7, 20, 28, 29]. 


\section{POLYMER-COATED COLLOIDS OR HAIRY SPHERES}

If the intermolecular attractive forces are strong enough, a colloidal suspension phaseseparates, or even flocculates or gels. As explained above, stability against flocculation may be ensured by steric or charge stabilization. In steric stabilization, the colloidal molecules are coated with grafted polymers - the "hair" - which can prevent particles from coming sufficiently close to experience a strong vdW-attraction.

However, changing the solvent or the temperature may turn the effective interaction from repulsion (HS-behavior) to attraction [32, 33, 34]. When sterically-stabilized colloidal particles are immersed in a good solvent for the polymer brushes, the solutes behave like HS, independently of temperature; this is the case, for example, of silica particles covered with a layer of octadecyl chains, when immersed in cycloexane. On the other hand, for each poor solvent there exists a Flory's theta-temperature $T_{\theta}$ [35], which is characteristic of the given solvent-polymer pair and has the following property: the solute particles behave like HS at $T>T_{\theta}$, whereas an attraction occurs at $T<T_{\theta}$. This occurs with e.g. silica particles with octadecyl chains, when dispersed in benzene. The term " $\theta$-solvent" indicates a poor solvent at $T=T_{\theta}$.

These effects originate from a competition between polymer-solvent and polymer-polymer interactions. First, the nature of the solvent influences the polymer conformation. In fact, in a good solvent the interactions between polymer elements - monomer units - and adjacent solvent molecules are strongly attractive and thus predominate over possible intra-chain polymer attractions. Consequently, the polymer will assume an "extended-chain" configuration, so as to reduce the number of intra-chain contacts between monomer units. Polymercoated colloidal particles will have fully-extended hair and thus the largest HS diameter possible, corresponding to the strongest solute-solute repulsion.

In a poor solvent, on the other hand, the polymer-solvent attractions are weak. Now it is the temperature that determines the solute-solute interaction. At $T>T_{\theta}$ the hair will be fully-extended, as in good solvents (HS behavior). At low temperatures $T<T_{\theta}$ the polymer segments find their own environment more satisfying than that provided by the solvent. This preference may produce more compact "globular" configurations, in which intra-chain polymer-polymer contacts occur more frequently ("curly hair"). In an alternative view, when two solute particles are in close contact, a high number of polymer-polymer attractions is 
favored by the interpenetration of the two polymeric layers.

In the literature on sterically-stabilized colloids [32, 33, 34], the attractive part of the potential for one-component hairy hard spheres (HHS) - due to the polymer-polymer interactions between surface layers of different particles - was described by a SW, with a depth proportional to the (maximum) overlap volume of the layers and temperature-dependent in analogy with the Flory-Krigbaum model for polymer segments [36]. The SW width equals the length of interpenetration of the stabilizing chains, whose maximum value coincides with the thickness $\ell$ of polymeric layer.

For mixtures, we could consider the most direct extension of the one-component SW model. In such a case, using Eq. (15), a SW potential could immediately be mapped onto a SHS one:

$$
t_{i j}^{\mathrm{eq}(\mathrm{SW})}=\left[\exp \left(\beta \epsilon_{i j}^{\mathrm{SW}}\right)-1\right] \frac{1}{3}\left[\left(1+\Delta_{i j}^{\mathrm{SW}}\right)^{3}-1\right] .
$$

When the SW is very narrow $\left(\Delta_{i j}^{\mathrm{SW}} \ll 1\right)$, one can approximate

$$
t_{i j}^{\mathrm{eq}(\mathrm{SW})} \approx\left[\exp \left(\beta \epsilon_{i j}^{\mathrm{SW}}\right)-1\right] \Delta_{i j}^{\mathrm{SW}}
$$

However, instead of a discontinuous SW model, we prefer to propose a potential with a similar but continuous attractive tail of finite range, i.e.,

$$
\phi_{i j}^{\mathrm{HHS}}(r)=\left\{\begin{array}{cc}
+\infty & r<\sigma_{i j} \\
-k_{B} T F(T) \rho_{\ell} V^{\mathrm{overlap}}\left(R_{i}+\ell, R_{j}+\ell, r\right) & \sigma_{i j} \leq r \leq \sigma_{i j}+2 \ell \\
0 & r>\sigma_{i j}+2 \ell,
\end{array}\right.
$$

where

$$
F(T)=\left\{\begin{array}{cc}
0 & T>T_{\theta} \\
T_{\theta} / T-1 & T<T_{\theta}
\end{array} .\right.
$$

Here, we call $F(T)$ Flory's temperature-function, and $\phi_{i j}^{\mathrm{HHS}}(r)$ is assumed to be proportional to the overlap volume between polymeric layers of the two HHS at separation $r$, with $\rho_{\ell}$ being a number density proportional to the polymer density in the stabilizing layer.

Within the linear approximation, one finds for the equivalent SHS model

$$
t_{i j}^{\mathrm{eq}(\mathrm{HHS})} \approx F(T) \frac{\eta_{\ell}}{2}\left[\frac{\sigma_{i} \sigma_{j}}{\sigma_{i j}^{2}}+\left(1+\frac{1}{4} \frac{\sigma_{i} \sigma_{j}}{\sigma_{i j}^{2}}\right) \frac{2 \ell}{\sigma_{i j}}+\frac{1}{2}\left(\frac{2 \ell}{\sigma_{i j}}\right)^{2}+\frac{1}{12}\left(\frac{2 \ell}{\sigma_{i j}}\right)^{3}\right],
$$


where $\eta_{\ell} \equiv \frac{\pi}{6} \rho_{\ell}(2 \ell)^{3}$. Since the thickness $\ell$ is much smaller than the particle sizes, one may expect - by analogy with the HS-depletion model - that the cubic approximation reads

$$
t_{i j}^{\mathrm{eq}(\mathrm{HHS})} \approx C_{1}\left[F(T) \eta_{\ell} \frac{\sigma_{i} \sigma_{j}}{\sigma_{i j}^{2}}\right]+C_{2}\left[F(T) \eta_{\ell} \frac{\sigma_{i} \sigma_{j}}{\sigma_{i j}^{2}}\right]^{2}+C_{3}\left[F(T) \eta_{\ell} \frac{\sigma_{i} \sigma_{j}}{\sigma_{i j}^{2}}\right]^{3} .
$$

Note that, since $F(T)=0$ when $T>T_{\theta}$, then $\lim _{T \rightarrow+\infty} F(T)=0$. Thus the form of $F(T)$ ensures that the high-temperature condition (10) is satisfied.

\section{SOLVATION FORCES. GURNEY-FRIEDMAN MODEL}

An indirect interaction between solute particles may also arise from solvation. To picture solvation effects, Gurney [37] and Frank and Evans [38] introduced the physically intuitive concept of cosphere or solvation layer. One supposes that any isolated solute particle is surrounded by some region in which the solvent has different properties than the bulk solvent, since its structure is markedly affected by the presence of the solute: some of the solventsolvent bonds have been broken by the introduction of the "foreign" particle. Clearly, such a region has no well-defined boundary, but Gurney's model assumes that significant effects come from only the few solvent molecules that are directly next to the solute particles, i.e., in a spherical shell whose thickness $\delta$ is taken to be a few solvent diameters or even the size of only one solvent molecule (for water, a molecular diameter of $2.76 \AA$ is acceptable). This

picture was first applied to electrolyte solutions by Friedman and coworkers [39]. In the ionic case, however, the previous definition of cosphere, with the same thickness for every ionic species, may be too restrictive, since the solvation region may extend even outside the cosphere, as occurs for very small ions $\left(\mathrm{Li}^{+}\right.$, and polyvalent ions such as $\mathrm{Mg}^{2+}, \mathrm{Ca}^{2+}$, etc.).

When two solute particles $i$ and $j$ approach sufficiently closely for their solvation layers to overlap, some of the cosphere solvent is displaced. Furthermore, the overlapping region contains solvent molecules which are now affected by the combined force field of two solutes, so that its structure might even differ from that of each isolated cosphere. The whole process, in which the sum of the cosphere volumes is reduced by overlap and the solvent relaxes to its normal bulk state, will be accompanied by a Gibbs free energy change. If the solvent molecules in the isolated solvation layers are in a state of lower free energy than those in the bulk, the overlap of two cospheres with the consequent expulsion of solvent gives rise to a free energy increase, and the resulting contribution to the interaction between two solutes 
is repulsive. When the solvent molecules in the solvation layers are in a state of higher free energy than those in the bulk, the expulsion of solvent from the overlapping region leads to a free energy decrease. In this case, both the free energy and the disruption of solvent structure are minimized when two solute particles $i$ and $j$ are brought close together, causing a net $i-j$ attraction.

Because of the lack of knowledge about the properties of the solvent in the solvation region, it is difficult to construct a detailed and physically sound microscopic model of the effects described above. Adopting a heuristic approach, Friedman and coworkers [39] proposed that the free energy change accompanying the cosphere overlapping of two HS solute particles $i$ and $j$ gives rise to the Gurney potential, defined by

$$
\phi_{i j}^{\text {Gurney }}(r)=A_{i j}(T, p) \frac{V^{\text {overlap }}\left(R_{i}+\delta, R_{j}+\delta, r\right)}{v_{0}} .
$$

Here the Gurney parameter $A_{i j}$ is in general a function of temperature $T$ and pressure $p$ and represents the molar free energy of transfer of solvent from the overlapping region of the $i-j$ cospheres to the bulk. As previously discussed, $A_{i j}<0$ corresponds to attraction. Furthermore, $v_{0}$ is the mean volume of a solvent molecule, while the volume of solvent returning to the bulk is given by the intersection volume of the cospheres surrounding the two solute HS at distance $r$, namely $V^{\text {overlap }}\left(R_{i}+\delta, R_{j}+\delta, r\right)$. The free-energy parameters $A_{i j}$ were determined numerically by fitting the model to experimental data.

The close resemblance of $\phi_{i j}^{\text {Gurney }}(r)$ to both $\phi_{i j}^{\mathrm{HHS}}(r)$ and $\phi_{i j}^{\mathrm{HS}-\mathrm{depl}}(r)$ is apparent. By analogy one obtains immediately

$$
t_{i j}^{\text {eq(Gurney-solvation })} \approx C_{1}\left[E_{i j}(T, p) \frac{\sigma_{i} \sigma_{j}}{\sigma_{i j}^{2}}\right]+C_{2}\left[E_{i j}(T, p) \frac{\sigma_{i} \sigma_{j}}{\sigma_{i j}^{2}}\right]^{2}+C_{3}\left[E_{i j}(T, p) \frac{\sigma_{i} \sigma_{j}}{\sigma_{i j}^{2}}\right]^{3} .
$$

where

$$
E_{i j}(T, p)=\frac{1}{2} \frac{\left|A_{i j}(T, p)\right|}{k_{B} T}\left[\frac{\frac{\pi}{6}(2 \delta)^{3}}{v_{0}}\right] .
$$

This expression may be applied, in particular, to both cases of solvation interactions hydrophobic bonding and interactions between reverse micelles - whose physical origin will be illustrated in the following.

\section{A. Hydrophobic interaction}

The hydrophobic interaction (or hydrophobic bonding) consists in the tendency shown by nonpolar portions of molecules or ions with long nonpolar chains or aromatic groups - 
for example, surfactants, phospholipids, glycerides, and dyestuffs - to aggregate in aqueous solutions, partially or completely removing such parts from contact with the solvent [2, 40, 41]. This hydrophobic attraction between nonpolar entities, which occurs exclusively in water, has - to a large extent - an entropic origin, related to the strong tendency of water molecules to form hydrogen bonds and associate [4].

The physical mechanisms underlying the solvation forces are rather well understood when the solvent is water. Polar molecules or polar groups of a solute feel strong attraction towards water molecules, and thus are said to be hydrophilic ("water-loving"). On the other hand, nonpolar molecules or nonpolar groups "dislike" water, and are called hydrophobic ("waterhating", or "water-avoiding").

The hydrophobic effect means that nonpolar particles have an extremely weak solubility in water, since inserting one of them - a noble gas atom, a chlorine or oxygen molecule, a hydrocarbon molecule, etc. - into water may actually lead to an increase of Gibbs free energy, i.e., $\Delta G_{\text {solution }}>0$. Indeed, the formation of a new cavity requires the breaking of many water-water hydrogen bonds with a considerable $\Delta G>0$, which cannot be compensated by the small $\Delta G<0$ provided by the new solute-water vdW interactions (the nonpolar solute cannot participate in the formation of hydrogen bonds). Then, in order to get a further decrease of $G$, the water molecules close to the solute reorient themselves, so as to create as many hydrogen bonds with adjacent water molecules as possible. The result is the formation of a "cage" - or hydration layer - around the solute, with more rigid water-water bonds than in the bulk. Such an additional ordering in the solvent, brought about by the introduction of a solute molecule, implies a significant entropy decrease, $\Delta S<0$, and thus a strong positive contribution $-T \Delta S$ to the total $\Delta G=\Delta H-T \Delta S$ of solution at constant $T$ and $p$. This explains why nonpolar particles are hydrophobic: one gets $\Delta G_{\text {solution }}>0$, when the entropic contribution dominates over the entalpic one $\Delta H$, which is usually small and may be positive or negative. On the other hand, at higher temperatures the solubility increases, and one may find $\Delta G_{\text {solution }}<0$. In fact, $\Delta S$ becomes much smaller, because the molecular thermal motion struggles more efficiently against the structure formation around a nonpolar solute.

The same hydrophobic effect is responsible for the above-mentioned hydrophobic bonding, where nonpolar parts of molecules or ions tend to aggregate. In fact, the solvent molecules prefer mutual contacts over those with the "foreign" substance (solute), while the aggre- 
gation of solutes reduces the total volume of their "cages", minimizing the loss of entropy. The hydrophobic interaction arises when overlap of hydration-layers occurs, and becomes increasingly attractive as the distance between two solute particles decreases.

This phenomenon in acqueous solutions of alcohols was studied by Friedman and Krishnan [42], who used a model potential containing a repulsive term of the $r^{-9}$ form, plus an attractive Gurney term given by Eq. (37), representing the overlap between cospheres. For the sustances they considered, these authors found values of the Gurney coefficient $A_{x x}$ in the range -190 to $-60 \mathrm{cal} \mathrm{mol}^{-1}$. The cosphere thickness $\delta$ was taken to be $2.76 \AA$, corresponding to one molecular layer of water.

Clark et al. [43] investigated the same physical systems with a more refined model, including a core potential based upon Lennard-Jones potentials for individual atom-atom interactions and again a Gurney term for the hydrophobic attraction. They found a minimum $\simeq-0.5 k_{B} T$ in their potentials of average force, that implies an overall tendency for those alcohols to associate when in an aqueous environment.

Hydrophobic bonding is very important in interface and colloid science. It is often the driving force behind the way in which biomolecules organize themselves and it is responsible for the formation of micelles, bimolecular layers, and lamellar structures.

\section{B. Reverse micelles in water-in-oil microemulsions}

Molecules having both hydrophilic and hydrophobic parts are said to be amphiphilic ("dual-loving", in the sense of being both "water-loving" and "water-hating"; from the Greek $\alpha \mu \varphi \iota=$ "on both sides"). An important example is provided by relatively short chain molecules with an ionizable or polar (thus hydrophilic) head-group and a nonpolar (thus hydrophobic) tail, consisting of one or several flexible hydrocarbon chain(s). Since these molecules can significantly lower the surface tension of a solution, they are generically called surfactants or surface-active agents [1, 2, 41]. When immersed in water, the head-group may become negatively or positively charged, or it remains polar with no net electric charge. Accordingly, the surfactants are classified as anionic, cationic or non-ionic.

Clearly, it is the hydrophilic head-group that keeps a surfactant solute dissolved in the water. The hydrophobic tail tends to avoid contact with water and to seek, as far as possible, a nonaqueous environment. The longer the hydrophobic tail, the poorer the solubility in water 
and, hence, the greater the tendency of the surfactant to escape from the acqueous solution. Consequently, as the solute concentration increases, a phase-separation may occur. Alternatively, the surfactant molecules accumulate at interfaces between water and other liquids or gases, or spontaneously self-assemble into supramolecular aggregates of mesoscopic size that minimize the number of contacts between water and hydrocarbon tails and maximize the number of tail-tail interactions.

If a surfactant is added to pure water under atmospheric pressure, its molecules first form a monolayer film at the water-air interface, with polar heads pointing towards the water and tails exposed to the air. Above a certain concentration of surfactant (critical micellization concentration), one finds an abrupt change in the properties of the solution; in fact, now the solute particles in the bulk begin to form supramolecular aggregates like micelles, planar lamellar bilayers, and vesicles, whose size and shape are, to a large extent, determined by the geometric properties of the surfactant molecules [1, 2]. These (direct) micelles have a nearly spherical structure, in which the head-groups are placed at the surface and are thus exposed to the aqueous environment, whereas the nonpolar tails occupy the interior of the micelle, avoiding any contact with water.

A surfactant, when added to a mixture of water and oil (an organic liquid immiscible with water), forms monolayers at every water-oil interface. Several disordered or partially ordered phases are possible, depending on temperature and surfactant concentration. In particular, one can get a microemulsion, which is a two-phase suspension of finely divided droplets of oil in water $(\mathrm{O} / \mathrm{W})$, or water in oil $(\mathrm{W} / \mathrm{O})$, depending on the relative concentration of the two liquids. Each droplet is coated with a monolayer film of surfactant, which separates water from oil. In W/O microemulsions one finds reverse (or inverted) micelles, where the core is formed by a droplet of water, and the surrounding surfactant molecules now have the headgroups inside the micelle, in contact with water, while their hydrocarbon tails point towards the outside oil phase [44, 45, 46, 47]. Clearly, such flexible tails resemble the polymer-hair of sterically stabilized colloids, but in reverse micelles the number of chains is lower and thus a large amount of oil can penetrate the surfactant-layer. For the sake of simplicity, we do not consider the possible presence of a cosurfactant, which is generally an alcohol and mixes with surfactant in the outside layer.

In most models for $\mathrm{W} / \mathrm{O}$ microemulsions the suspending medium, containing mainly oil, is treated as a continuous phase, and the reverse micelles are represented as composed of 
two parts. The internal one, including both the water droplet and surfactant head-groups, is assumed to be a spherical and impenetrable core, with HS radius $R$. The external part consists of a concentric, penetrable spherical layer, with thickness equal to the length $\ell$ of the aliphatic chains of surfactant in their fully-extended conformation. Thus the total radius of a micelle is $R^{\prime}=R+\ell$. Because of the flexibility of the chains, $\ell$ has sometimes been allowed to depend on temperature, i.e., $\ell=\ell(T)$ [46].

The short-ranged attraction between reverse micelles seems to be mainly determined by the overlapping of the penetrable surfactant-layers during collisions [44, 45, 47]. The interpenetration of the aliphatic chains of the surfactant induces oil removal. Now, the partial molar volume of oil inside the surfactant-layer is expected to be larger than in the pure oil-phase (this effect is related to the volume of $\mathrm{CH}_{2}$ and $\mathrm{CH}_{3}$ groups in the aliphatic layers). A difference of $0.2 \AA^{3}$ seems to be sufficient to explain an interaction potential compatible with light scattering experiments [45].

Roux and Bellocq [45] proposed the simplest model for equal-sized micelles, assuming that the interaction potential is proportional to the overlap volume. The extension of their formula to mixtures is immediate. According to our terminology, the resulting expression is equivalent to a particular Gurney potential, i.e.,

$$
\phi_{i j}^{\text {rev.micel. }}(r)=\left\{\begin{array}{cc}
+\infty & r<\sigma_{i j} \\
-k_{B} T \Delta \rho V^{\text {overlap }}\left(R_{i}+\ell, R_{j}+\ell, r\right) & \sigma_{i j} \leq r \leq \sigma_{i j}+2 \ell \\
0 & r>\sigma_{i j}+2 \ell,
\end{array}\right.
$$

where $\Delta \rho$ is an adjustable parameter which depends only on the oil.

More refined models also include a Hamaker-vdW attraction between water cores. Electrostatic contributions and other more complicated terms have not been considered in the present paper.

\section{CONCLUSIONS}

We have started from the problem of building up statistical mechanical models for fluid mixtures of mesoscopic particles, like colloids, micelles or globular proteins, by using very simple effective potentials containing a HS repulsion plus a short-ranged attractive tail that represent the interaction between big particles after averaging out the microscopic degrees of freedom related to much smaller molecules (solvent, added polymers, etc.). The simplest 
tail corresponds to the highly idealized surface adhesion, modeled through a $\delta_{+}$-term, of the SHS potential. Since we are interested in multicomponent SHS fluids, and in the past difficulties had been encountered in choosing their stickiness parameters $t_{i j}$, the present paper has focused on the relationship between $t_{i j}$ and particle sizes, i.e., on the possible functional forms of $t_{i j}\left(T, \sigma_{i}, \sigma_{j}\right)$. To elucidate this issue we have regarded the SHS potentials as models that may be derived, with some simplification, from more realistic interactions. The idea of associating an equivalent SHS representation to a realistic interaction, by requiring the equality of the second virial coefficients, is already known and widely accepted. We have chosen this mapping based upon the virial equivalence because of its simplicity and partial analytical tractability. Since $B_{2}$ yields only the first correction of pressure with respect to ideality, one can reasonably expect that the performance of the resulting SHS model should worsen with increasing density. By requiring the equivalence of quantities different from $B_{2}$, one could obtain alternative mappings and generally different values for the effective parameters, leading to more successful results at high densities. An application of such an idea was given for example in Ref. [47], where the solvent molecules were modeled as SHS by requiring the equivalence of structural properties, i.e. the solvent-solvent structure factor and the coordination number. Unfortunately, this approach does not admit an analytical treatment, and a comparison with it goes beyond the scope of the present paper.

Our aim was to investigate the most interesting cases of short-ranged attractive interactions for some paradigmatic physical systems. To provide a physically sound basis for the choice of $t_{i j}$ and, in particular, its dependence on the particle diameters, we have presented a detailed and self-contained overview on several topics related to short-ranged attractive interactions in real mixtures of neutral mesoscopic particles. We have considered: i) the van der Waals or dispersion forces - direct interactions - and three cases with indirect, solvent-mediated, attractions: ii) depletion forces, iii) polymer-coated colloids, and iv) solvation forces (in particular, hydrophobic bonding and interactions between reverse micelles in water-in-oil microemulsions).

Due to obvious analytical difficulties, our analysis has been restricted to the determination of the leading terms of $t_{i j}$. These have been evaluated by series expansion of the Mayer function that appears in the second virial coefficient. We have discussed in particular the linear, quadratic and cubic approximations and their respective ranges of validity.

The main result is that - in almost all cases considered - the leading contributions to $t_{i j}$ 
can be expressed as

$$
t_{i j}^{\mathrm{eq}(\mathrm{tail})} \approx C_{1}\left[M_{i j}(X, \ldots) \frac{\sigma_{i} \sigma_{j}}{\sigma_{i j}^{2}}\right]+C_{2}\left[M_{i j}(X, \ldots) \frac{\sigma_{i} \sigma_{j}}{\sigma_{i j}^{2}}\right]^{2}+\cdots,
$$

where - in most scenarios - $X$ is the temperature $T$, and the property

$$
\lim _{T \rightarrow+\infty} M_{i j}(T, \ldots)=0
$$

ensures that the high-temperature condition of Eq. (10) is satisfied. In the case of the depletion attraction, $X$ coincides with the packing fraction $\eta_{0}$ of the depletant particles. For hairy HS, both $T$ and $\eta_{\ell}$ are variables included in $M_{i j}$. As regards the dependence of $t_{i j}$ on the big particle sizes - which was the basic question of the present work - , it is remarkable that $t_{i j}$ can be expressed as a simple polynomial in the variable $\left(\sigma_{i} \sigma_{j} / \sigma_{i j}^{2}\right)$. A quadratic approximation may already be sufficient. The only case in which the expression for $t_{i j}$ differs from that given in Eq. (41) is the Sutherland model for polarizable HS, which yields

$$
t_{i j}^{\mathrm{eq}(\mathrm{PHS})}=12 \frac{T_{\mathrm{H}}}{T}\left(\frac{\sigma_{i} \sigma_{j}}{\sigma_{i j}^{2}}\right)^{3}+15\left[\frac{T_{\mathrm{H}}}{T}\left(\frac{\sigma_{i} \sigma_{j}}{\sigma_{i j}^{2}}\right)^{3}\right]^{2}+8\left[\frac{T_{\mathrm{H}}}{T}\left(\frac{\sigma_{i} \sigma_{j}}{\sigma_{i j}^{2}}\right)^{3}\right]^{3} .
$$

It is pleasant that even here we find powers of the same basic size-dependent factor, $\left(\sigma_{i} \sigma_{j} / \sigma_{i j}^{2}\right)$. Note that this factor has the property that the sticky attraction vanishes when at least one of the two particles $i$ and $j$ becomes a point, i.e., it satisfies the point-limit condition

$$
\lim _{\sigma_{i} \text { or } \sigma_{j} \rightarrow 0} t_{i j}\left(T, \sigma_{i}, \sigma_{j}\right)=0 .
$$

This condition would be expected to hold for any interaction of "adhesive" type which in the limit involves a particle surface of vanishing area [48].

The similarity among most of the resulting expressions for $t_{i j}^{\mathrm{eq}(\mathrm{tail})}$ can easily be understood. In fact, most of the different short-ranged attractions considered here have been explained - by different authors - in terms of quite similar models, where mesoscopic particles are represented as hard spheres with a surrounding, concentric spherical layer (see Figure 1). In the AO case this layer is characterized somewhat indirectly by the fact that the density of the centers of mass of the depletant polymers is zero inside the layer but has a nonzero value outside it. In the other cases, the layer has a clearer physical reality. For polymer-coated colloids, for example, the layer is the polymeric film grafted on the colloidal surface. In the hydrophobic bonding the layer is formed by the solvation water molecules. 
For reverse micelles, the core comprising the water droplet and the polar heads of surfactants is surrounded by a layer made up of hydrocarbon tails of surfactants plus a certain quantity of oil.

It is very appealing, and somewhat surprising, that the factor $\sigma_{i} \sigma_{j} / \sigma_{i j}^{2}$ appears even in our result for the Hamaker potential, which refers to a direct interaction where no spherical layer around the solutes is involved. Note that this dependence on the particle diameters is clearly due to the Hamaker integration, since for point-dipoles at the centre of hard spheres (polarizable HS) we have found a different factor, i.e., $\left(\sigma_{i} \sigma_{j} / \sigma_{i j}^{2}\right)^{3}$.

We remark that the models we have considered can be divided into two different classes. The first one includes the two models of dispersion forces (Hamaker potential and polarizable HS), with the common feature of having an attractive $r^{-6}$ tail. The second class refers to the solvent-mediated attractions (depletion effects, polymer-coated colloids, solvation forces). Here, we have reported the simplest examples, which can be regarded as variants of one single model: hard spheres with a penetrable concentric spherical layer ('cosphere', in a wider sense). As a consequence, since the attraction depends on the volume overlap of the cospheres, the potentials of all these models are 'truncated', i.e. they are rigorously zero beyond some characteristic distance.

The main difference between the above mentioned classes - infinite tail in the first, finite tail in the second - might suggest that the idea of representing realistic potentials by an equivalent SHS model is justifiable for the second class, but somewhat more questionable when the tail is infinite. In particular, since a proper treatment of long tails is essential for thermodynamics, the SHS-mapping of the Hamaker and Sutherland potentials might introduce some qualitative differences in such a kind of properties. This viewpoint is certainly correct and Hamaker and Sutherland potentials should be appropriately distinguished from the remaining models of this paper. In fact, an 'exact' treatment of all these models would surely yield very different thermodynamic and structural predictions. Nevertheless, in our context the $B_{2}$-mapping onto SHS can be expected to yield a representation of realistic interactions that is simple, analytically tractable, and reliable in appropriate regimes, at low and intermediate densities.

In a companion paper [49], we have applied a perturbative approach to the solution of the polydisperse SHS model within the Percus-Yevick approximation. The suggestions put forward in the present paper regarding the relationship between stickiness and size, could 
help to improve the necessary input to that kind of scheme.

In conclusion, the present paper suggests - for multicomponent SHS models - the expression for $t_{i j}$ given by Eq. (41) as a simple choice that is physically justified by its relation to the above-mentioned models of real interactions. Clearly, Eq. (41) is an approximate result, but we believe that it correctly includes the leading terms of the dependence of $t_{i j}$ on the particle sizes. In spite of the rather drastic approximations used here, this could be useful with the rationale of having a simple and tractable representation of rather complex interactions, at the simplest possible level of description.

\section{Acknowledgments}

We are grateful to Andrés Santos and Saïd Amokrane for enlightening discussions and critical reading of the manuscript. The work in Italy was supported by a MIUR-COFIN 20062007 grant (Colloidal mixtures, globular proteins and liquid crystal-like phases of biopolymers).

\section{APPENDIX A: VOLUME OVERLAP BETWEEN SPHERES}

The volume of the intersection between HS with radii $a$ and $b$, at distance $r$, is

$V^{\text {overlap }}(a, b, r)=\left\{\begin{array}{cc}(4 \pi / 3) \min \left(a^{3}, b^{3}\right) & 0<r<|a-b|, \\ \frac{\pi}{12}\left[-3\left(a^{2}-b^{2}\right)^{2} \frac{1}{r}+8\left(a^{3}+b^{3}\right)-6\left(a^{2}+b^{2}\right) r+r^{3}\right] & |a-b|<r<a+b, \\ 0 & r>a+b .\end{array}\right.$

For $|a-b|<r<a+b$ this expression can conveniently be rewritten as

$$
V^{\text {overlap }}(a, b, r)=\frac{\pi}{12}\left[12 a b(r-a-b)^{2}+4(a+b)(r-a-b)^{3}+(r-a-b)^{4}\right] \frac{1}{r} .
$$

Taking $a=\sigma_{i 0}=\left(\sigma_{i}+\sigma_{0}\right) / 2$ and $b=\sigma_{0 j}=\left(\sigma_{j}+\sigma_{0}\right) / 2$, one gets $V^{\text {overlap }}\left(\sigma_{i 0}, \sigma_{j 0}, r\right)=$ $V_{i j}^{\text {overlap }}(r)$ of Eq. (29). 
[1] J. L. Barrat, and J. P. Hansen, Basic Concepts for Simple and Complex Liquids (Cambridge University Press, Cambridge) (2003).

[2] J. Lyklema, Fundamentals of Interface and Colloid Science, Vol 1 (Academic Press, London) (2000).

[3] D. Frenkel, in Soft and Fragile Matter. Nonequilibrium Dynamics, Metastability and Flow, ed. M. E. Cates and M. R. Evans (SUSSP Publications, Edinburg, and Institute of Physics Publishing, London) (2000).

[4] M. Kleman, and O. D. Lavrentovich, Soft Matter Physics: An Introduction (Springer-Verlag, New York) (2003).

[5] J. P. Hansen and I. R. Mc Donald, The Theory of Simple Liquids (Academic, London) (1986).

[6] S. Amokrane, A. Ayadim, P. Germain, J. G. Malherbe, and C. Regnaut, in Trends in Chemical Physics Vol. 11, 119-142 (2004).

[7] A. A. Louis, E. Allahyarov, H. Löwen, and R. Roth, Phys. Rev. E 65, 061407 (2002).

[8] B. Widom, Statistical Mechanics. A Concise Introduction for Chemists (Cambridge University Press, Cambridge) (2002) p. 98.

[9] H. L. Friedman, A Course in Statistical Mechanics (Prentice-Hall, Englewood Cliffs, New Jersey) (1985) p. 135.

[10] J. O. Hirschfelder, C. F. Curtiss, and R. B. Bird, Molecular Theory of Gases and Liquids (John Wiley \& Sons, Inc.) (1965).

[11] Note that, as a consequence of the two different definitions of short-ranged forces, the classical Lennard-Jones (LJ) potential - with $r^{-6}$ tail - should be considered short-ranged according to Widom's definition, but long-ranged according to Hirschfelder's one. In our opinion, this discrepancy in terminology at the level of potentials may be disconcerting but is not essential, while the consequent differences at the level of mechanical-statistical properties should be emphasized. In fact, fluids with potentials "decaying exponentially or faster" with $r$ may exhibit undoubtly different physical behaviors compared to systems characterized by longerranged $r^{-n}$ potentials (like LJ). This can be argued by considering, for instance, the wetting at attractive walls, as well as the asymptotic decay of their pair correlation function at large distances. 
[12] R. J. Baxter, J. Chem. Phys. 49, 2270 (1968). R. J. Baxter, in: Physical Chemistry, an Advanced Treatise, Vol. 8A, ed. D. Henderson (Academic Press, New York, 1971) ch. 4. R. O. Watts, D. Henderson and R. J. Baxter, Advan. Chem. Phys. 21, 421 (1971).

[13] J. W. Perram and E. R. Smith, Chem. Phys. Lett. 35, 138 (1975).

[14] B. Barboy and R. Tenne, Chem. Phys. 38, 369 (1979).

[15] G. Stell, J. Stat. Phys. 63, 1203 (1991).

[16] C. Regnaut and J. C. Ravey, J. Chem. Phys. 91, 1211 (1989).

[17] A. Jamnik, (a) J. Chem. Phys. 105, 10511 (1996). (b) J. Chem. Phys. 114, 8619 (2001).

[18] A. Santos, S. Bravo Yuste, and M. L. De Haro, J. Chem. Phys. 109, 6814 (1998).

[19] M. A. Miller, and D. Frenkel, Phys. Rev. Lett. 90, 135702 (2003). M. A. Miller, and D. Frenkel, J. Chem. Phys. 121, 535 (2004). M. A. Miller, and D. Frenkel, J. Phys. Cond. Mat. 16, S4901 (2004).

[20] C. Regnaut, S. Amokrane, and Y. Heno, J. Chem. Phys. 102, 6230 (1995).

[21] D. Gazzillo, and A. Giacometti, J. Chem. Phys. 113, 9837 (2000). Physica A, 304, 202 (2002).

[22] D. Gazzillo, and A. Giacometti, Mol. Phys. 100, 3307 (2002).

[23] D. Gazzillo, and A. Giacometti, Mol. Phys. 101, 2171 (2003). J. Appl. Crystal. 36, 832 (2003).

[24] D. Gazzillo, and A. Giacometti, J. Chem. Phys. 120, 4742 (2004).

[25] R. Fantoni, D. Gazzillo, and A. Giacometti, J. Chem. Phys. 122, 034901 (2005). Phys. Rev. E 72, 011503 (2005).

[26] Taking the limit $w \rightarrow 0$ of the function $\varphi_{w}(x)=w^{-1}$ for $x_{0}<x<x_{0}+w$, and $=0$ elsewhere, one gets the asymmetric Dirac delta distribution $\delta_{+}(x)$, which has the property: $\int_{A}^{B} d x F(x) \delta_{+}\left(x-x_{0}\right)=F\left(x_{0}^{+}\right)$, if $A \leq x_{0}<B$, and $=0$, if $x_{0}<A$ or $x_{0} \geq B$.

[27] H. C. Hamaker, Physica 4, 1058 (1937).

[28] S. Amokrane, J. Chem. Phys. 108, 7459 (1998).

[29] J. C. Malherbe, C. Regnaut, and S. Amokrane, Phys. Rev. E 66, 061404 (2002).

[30] S. Asakura, and F. Oosawa, J. Chem. Phys. 22, 1255 (1954). S. Asakura, and F. Oosawa, J. Polym. Sci. 33, 183 (1958).

[31] A. Vrij, Pure Appl. Chem. 48, 471 (1976).

[32] C. G. de Kruif, P. W. Rouw, W. J. Briels, M. H. G. Duits, A. Vrij, and R. P. May, Langmuir 5, 422 (1989).

[33] H. Verduin, and J. K. G. Dhont, J. Colloid Interface Sci. 172, 425 (1995). 
[34] M. H. G. Duits, R. P. May, A. Vrij, and C. G. de Kruif, Langmuir 7, 62 (1991).

[35] P. J. Flory, Principles of Polymer Chemistry (Cornell University Press, Ithaca) (1953).

[36] P. J. Flory, and W. R. Krigbaum, J. Chem. Phys. 18, 1086 (1950).

[37] R. W. Gurney, Ionic Processes in Solution, Dover, New York (1953).

[38] H. S. Frank, and M. W. Evans, J. Chem. Phys. 13, 507 (1945).

[39] P. S. Ramanathan, and H. L. Friedman, J. Chem. Phys. 54, 1086 (1971). H. L. Friedman, and C. V. Krishnan, in Water, A Comprehensive Treatise, Vol. 3, ed. F. Franks (Plenum Press, New York) (1973). H. L. Friedman, and W. D. T. Dale, in Statistical Mechanics, Part A: Equilibrium Techniques, ed. B. J. Berne (Plenum Press, New York) (1977).

[40] F. Franks, in Water, A Comprehensive Treatise, Vol. 4, ed. F. Franks (Plenum Press, New York) (1978).

[41] A. Ben-Naim, Hydrophobic Interactions (Plenum Press, New York, 1980).

[42] H. L. Friedman, and C. V. Krishnan, in The Physical Chemistry of Aqueous Systems, ed. R. L. Kay (Plenum Press, New York) (1973).

[43] A. H. Clark, F. Franks, M. D. Pedley, and D. S. Reid, J. Chem. Soc. Faraday Trans. 1, 73, 290 (1977).

[44] B. Lemaire, P. Bothorel, and D. Roux, J. Phys. Chem. 87, 1023 (1983). S. Brunetti, D. Roux, A. M. Bellocq, G. Fourche, and P. Bothorel, 87, 1028 (1983).

[45] D. Roux, and A. M. Bellocq, in Physics of Amphiphiles: Micelles, Vesicles, and Microemulsions, ed. V. Degiorgio and M. Corti, p. 842 (Academic Press, New York) (1985).

[46] M. Bouaskarne, S. Amokrane, and C. Regnaut, J. Chem. Phys. 114, 2442 (2001).

[47] S. Amokrane, and C. Regnaut, J. Chem. Phys. 106, 376 (1997).

[48] B. Barboy, Chem. Phys. 11, 357 (1975).

[49] R. Fantoni, D, Gazzillo, A. Giacometti, and P. Sollich J. Chem. Phys. 125, 164504 (2006). 


\begin{tabular}{lccc}
\hline Approximation & $\lambda=0.1$ & $\lambda=0.2$ & $\lambda=0.3$ \\
\hline linear & 6300 & 1800 & 700 \\
quadratic & 1050 & 300 & 117 \\
cubic & 630 & 180 & 70 \\
\hline
\end{tabular}

TABLE I: Approximate lower bound $T^{\mathrm{min}} / K$ for the applicability of the linear, quadratic, cubic approximation to the Mayer function $f_{i j}$, as a function of the parameter $\lambda=\sigma_{0} / \sigma$ (see text).

\begin{tabular}{lcccccc}
\hline Approximation & $\lambda=0.1$ & $\lambda=0.3$ & $\lambda=0.5$ & $\lambda=0.7$ & $\lambda=0.9$ & $\lambda=1.0$ \\
\hline linear & 0.006 & 0.017 & 0.025 & 0.03 & 0.0375 & 0.04 \\
quadratic & 0.04 & 0.10 & 0.15 & 0.19 & 0.23 & 0.24 \\
cubic & 0.06 & 0.17 & 0.25 & 0.32 & 0.38 & 0.4 \\
\hline
\end{tabular}

TABLE II: Approximate upper bound $\eta_{0}^{\max }$ for the applicability of the linear, quadratic, cubic approximation to the Mayer function $f_{i j}$, as a function of $\lambda$ defined above. 

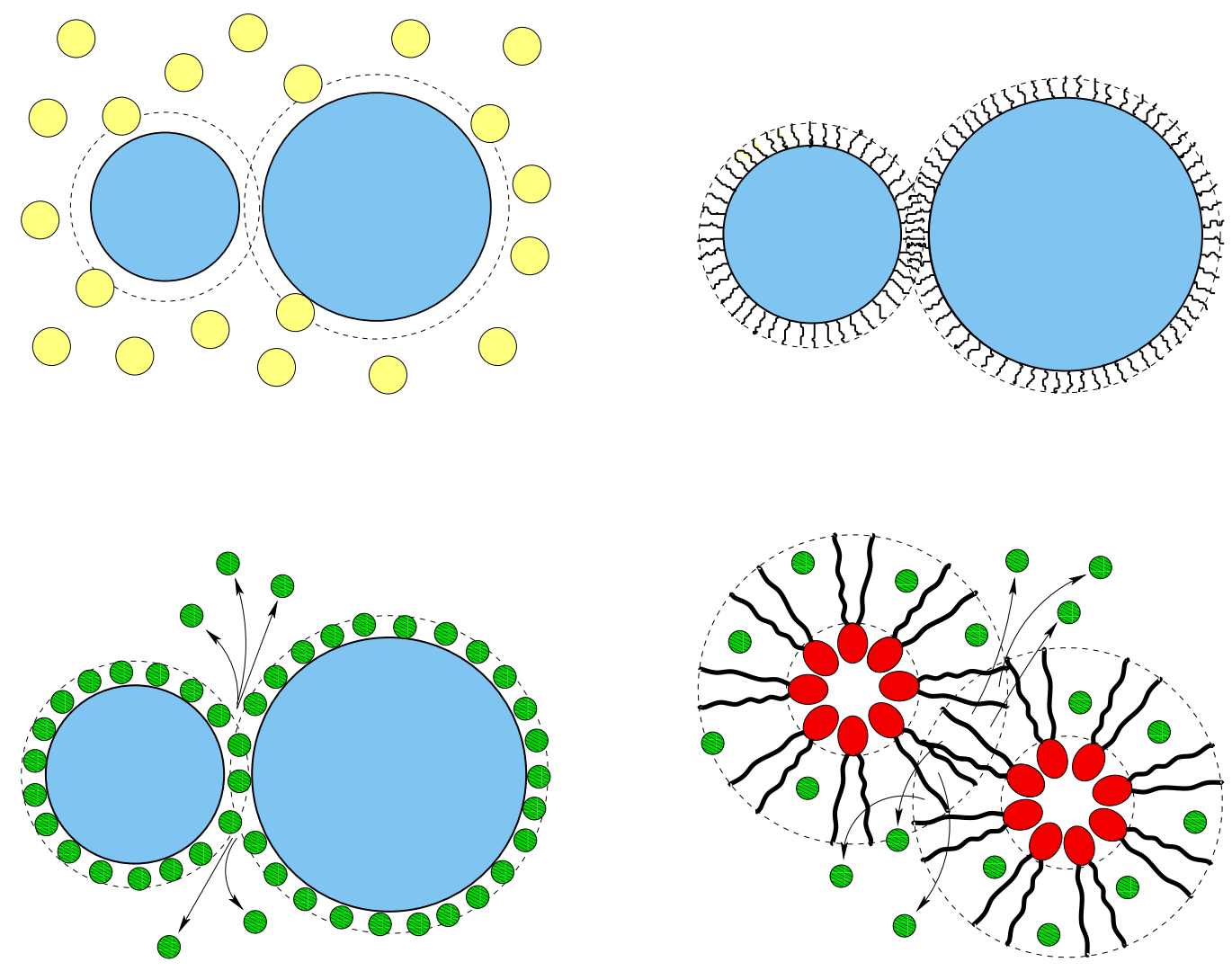

FIG. 1: Schematic representation of some systems described by SHS equivalent models in the text. Upper left panel: Excluded-volume depletion attraction between big spheres (solutes) in a sea of smaller depletant particles (light-grey). The dashed curves represent the excluded-volume regions where the centers of the depletant particles cannot penetrate. Upper right panel: Polymer-coated colloids or hairy spheres, when their sterically-stabilizing layers of grafted polymers overlap. Lower left panel: Overlap of solvation layers ('cospheres') in the Gurney-Friedman model, and expulsion of solvent from the overlapping region. The small spheres (dark-grey) represent solvent molecules. Lower right panel: Interaction between reverse micelles in water-in-oil microemulsions. In each micelle the internal dashed curve indicates the impenetrable core, formed by a droplet of water where the head-groups of the surfactant molecules are immersed. The region between the core and the external dashed curve is the penetrable part of the micelle, corresponding to the hydrocarbon tails and containing some oil molecules (small spheres). The micellar attraction is mainly due to oil removing from the overlapping region and its transfer to the bulk. 